Cahiers du MONDE RUSSE

\section{Cahiers du monde russe}

Russie - Empire russe - Union soviétique et États indépendants

$53 / 4 \mid 2012$

Varia

\title{
Une philosophie dans les marges
}

Le cas du conceptualisme moscovite

Philosophy at the margins. The case of Moscow Conceptualism

\section{Emanuel Landolt et Michail Maiatsky}

\section{(2) OpenEdition}

1 Journals

Édition électronique

URL : http://journals.openedition.org/monderusse/9402

DOI : $10.4000 /$ monderusse. 9402

ISSN : $1777-5388$

Éditeur

Éditions de l'EHESS

\section{Édition imprimée}

Date de publication : 15 décembre 2012

Pagination : $571-591$

ISSN : $1252-6576$

\section{Référence électronique}

Emanuel Landolt et Michail Maiatsky, «Une philosophie dans les marges », Cahiers du monde russe [En ligne], 53/4 | 2012, mis en ligne le 10 décembre 2015, Consulté le 26 avril 2019. URL : http:// journals.openedition.org/monderusse/9402 ; DOI : 10.4000/monderusse.9402 


\title{
UNE PHILOSOPHIE DANS LES MARGES
}

\author{
Le cas du conceptualisme moscovite
}
L'atelier est peut-être le seul endroit où souffle un vent de liberté. Viktor Pivovarov

La philosophie soviétique n'occupera probablement pas une place de choix au panthéon de la philosophie mondiale. Non seulement, aux yeux du grand public elle reste suspecte de compromission idéologique mais, de plus, elle a été sacrifiée sur l'autel de l'oubli de la période soviétique, son refoulement permettant la construction d'une philosophie postsoviétique. La philosophie de l'époque soviétique semble relever des éléments les plus rébarbatifs de cette civilisation. En effet, même les rares spécialistes occidentaux qui, guerre froide oblige, se sont penchés sur ce phénomène ${ }^{1}$, n'ont pas manqué de s'interroger : « N'a-t-on pas perdu notre temps ? $\gg^{2}$. Les auteurs, en Russie comme à l'étranger ${ }^{3}$, qui lui consacrent des livres, et leurs lecteurs ne continuent-ils pas à perdre leur temps ? Comment expliquer, justifier - pardonner ? - l'intérêt que suscite encore et à nouveaux frais la «philosophie soviétique »? C'est afin de démontrer l'existence d'une philosophie ou tout du moins d'une pensée à l'époque soviétique tardive, grosso modo la période brejnévienne, que nous avons choisi d'examiner ici un « cas-limite », celui

1. Voir la revue Studies in Soviet Thought (devenue ensuite Studies in East European Thought) et la série des livres « Sovetica » qui y ont été édités.

2. Jósef M. Bocheński, « Did we not waste our time ? », Studies in Soviet Thought, 42 (3), 1991, p. 295-302.

3. Nous pensons ici à la somme d'Evert van der Zweerde, Soviet Philosophy - the Ideology and the Handmaid : a historical and critical analysis of Soviet philosophy, with a case-study into Soviet history of philosophy, Nijmegen : University of Nijmegen, 1994, qui est une tentative de réponse historico-critique à la question de la philosophie soviétique, mais aussi une mise en exergue originale de la tendance hégélienne qui prédominait dans l'approche de l'histoire de la philosophie en Union soviétique. Voir plus en détail du même auteur : Soviet historiography of philosophy : istoriko-filosofskaja nauka, Dordrecht - Boston : Kluwer, 1997. 
du conceptualisme moscovite. Mais pour ce faire, il nous faut d'abord approcher, circonscrire, la notion de philosophie russe puis soviétique.

À côté des discussions d'ampleur nationale sur ce qu'était la « littérature soviétique », les débats sur la philosophie paraissent confidentiels. Si la littérature d'avant et d'après la période soviétique ne pose aucun problème définitionnel, et que l'on peut facilement fixer un cadre à la philosophie soviétique, il n'est pas toujours évident de circonscrire ce que peut être une philosophie russe ${ }^{4}$. Lorsque la page s'est définitivement tournée en 1991, la philosophie, redevenue russe après avoir été longtemps soviétique, s'interrogea sur son destin. Fallait-il reprendre la tradition interrompue, la «nôtre », celle de Vladimir Solov'ev, Nikolaj Berdjaev, Sergej Bulgakov, Pavel Florenskij ? Ou fallait-il au contraire renoncer à un tel passéisme et profiter du changement de cap pour renouer avec la philosophie contemporaine mondiale ? Mais dans ce cas, avec laquelle précisément ? Peu à peu ces atermoiements se sont cristallisés en une conviction chez les philosophes ayant émergé après la perestroïka : on ne pouvait pas prétendre que l'histoire s'était arrêtée il y a soixante-dix ans et supposer que, pendant tout ce temps, le pays n'avait été en aucune manière un lieu de pensée, en particulier au cours de la période qui nous occupe ici, celle des années $1970^{5}$.

Après le dégel et le durcissement idéologique qui lui succède, des changements s'opèrent progressivement dans le champ philosophique. Les uns, basés sur une confrontation directe avec la philosophie officielle, se sont soldés par des licenciements brutaux, des interdictions d'enseigner, des traitements psychiatriques forcés, des carrières et des vies brisées, des suicides. D'autres, au contraire, se sont produits sans confrontation directe, mais sciemment ou spontanément, par une voie de contournement. Les personnes concernées ont renoncé, parfois tout à fait sincèrement, à la prétention d'intervenir dans le domaine surprotégé de la philosophie, et ont obtenu une liberté incomparablement plus grande que celle à laquelle elles auraient pu prétendre en s'y maintenant. Sans heurter les censeurs vigilants de l'idéologie, elles ont travaillé, agi et écrit en tant que scientifiques, méthodologues, écrivains, artistes, metteurs en scène, pédagogues, en exerçant ce qui, en d'autres circonstances sociales et culturelles, aurait pu parfaitement être considéré comme de la philosophie, ou encore en pratiquant un intense échange d'idées avec des philosophes.

Il est important de tenir compte de ces faits pour comprendre les raisons, historiquement contingentes, qui ont influé sur la répartition des tâches et des gens entre la philosophie et les autres activités intellectuelles, scientifiques ou non. «Pensée », terme a priori plus vague, se montre plus opérationnel pour donner de la forme

\footnotetext{
4. De nombreux livres et numéros spéciaux ont été consacrés à ce sujet qui n'est pas le nôtre ici (voir M. Soboleva, éd., Rossijskaja postsovetskaja filosofija: opyt samoanaliza [Philosophie russe postsoviétique : tentative d'auto-analyse], München - Berlin : Otto Sagner, 2009 [= Specimina Philologiae Slavicae, Bd. 154].

5. Voir en particulier le livre du philosophe Mihail Ryklin, Svoboda i zapret : kul'tura v epohu terrora [Liberté et interdit : la culture à l'époque de la terreur], M. : Logos, 2008, qui traite sur le mode du témoignage cette question des lieux de la pensée en Union soviétique.
} 
aux processus intellectuels de l'époque. L'ambivalence supplémentaire soviétique consistait en ce que la philosophie était un lieu privilégié et, dès lors, très contrôlé. Ceux qui ne tenaient pas absolument à l'appellation ont préféré échapper tant au contrôle qu'aux stigmates du nom. Jamais l'idée ne serait venue ni à M. Bahtin, ni à Yu. Lotman de se qualifier de philosophe, alors qu'ils seront plus tard identifiés comme tels par des acteurs de la philosophie postsoviétique comme Mihail Ryklin ou encore Natalja Avtonomova, puis officiellement sacrés philosophes : un volume entier de l'imposante série «Philosophes en Russie de la seconde moitié du $\mathrm{xx}^{\mathrm{e}}$ siècle » est dévolu à chacun des deux ${ }^{6}$.

\section{La philosophie soviétique, des velléités purement scientifiques?}

Qu'il soit lié aux sciences dures ou à une « logique dialectique » érigée en une science supérieure, le profil scientifique que voulait se donner la philosophie en URSS aura probablement induit en erreur les historiens de la période, les poussant ainsi à ne chercher les interactions de la philosophie qu'avec les sciences et éclipsant le rôle des pratiques artistiques comme partenaire et interlocuteur de la philosophie. Nous évoquerons ici un épisode crucial de l'échange entre la philosophie et l'art dans la dernière décennie de l'existence du régime et de la philosophie soviétiques. Dans les marges de l'histoire de la philosophie soviétique, et dans une apparente indépendance par rapport à celle-ci, se laissent lire d'autres projets collectifs et singuliers qui révèlent que, sous le réseau officiel de la philosophie académique et comme en parallèle à celle-ci, à son insu, se dessine une mosaïque de penseurs aux parcours curieux, connectés les uns aux autres par un réseau souterrain d'amitiés ou, pour reprendre les mots d'Il'ja Kabakov, par un destin commun. Cette foule bigarrée trouve aussi ses lieux privilégiés de réunion et d'échange : ce sont des espaces intimes, des caves, des cuisines, des greniers, des ateliers semi-clandestins, dans lesquels évolue une nébuleuse d'artistes, penseurs, philosophes, poètes, plasticiens, performers, rassemblés sous l'étiquette de conceptualisme moscovite ${ }^{7}$. Cette appellation, qui ne rend pas complètement compte de la diversité des courants et des personnes qui en composent le tissu humain, recouvre moins une école ou une institution qu'un réseau d'amitiés basé dans un cadre privé, avec des pratiques codifiées et régulières prenant la forme d'exposition d'œuvres,

6. V.L. Mahlin, éd., Mihail Mihajlovič Bahtin, M. : ROSSPĖN, 2010 ; V. Kantor, éd., Jurij Mihajlovič Lotman, M. : ROSSPĖN, 2009.

7. Nom inventé par Boris Groys dans un article publié en 1979 dans la revue artistique de l'émigration A-Ya créée par le sculpteur Šelkovskij : B. Groys, « Moskovskij romantičeskij konceptualizm [Le conceptualisme romantique moscovite] », réédité dans B. Groys, Isskustvo utopii [L'art de l'utopie], M. : Hudožestvennij žurnal, 2003, p. 168-186. Il s'agit d'un mouvement artistique russe, né à Moscou au début des années 1970, et dont les membres sont encore actifs aujourd'hui. Afin de ne pas confondre les différentes périodes, nous nous concentrerons surtout dans la première partie sur l'époque qui précède l'éclosion réelle de ce mouvement, peu explorée jusqu'à aujourd'hui et que l'on peut situer, selon le journal de Kabakov, vers la fin des années 1960 et le début des années 1970. 
de lectures poétiques, de conférences privées, etc. Cet espace intérieur, pour ainsi dire débarrassé des contraintes institutionnelles propres aux académies et institutions de toute sorte, permettait ainsi la mise en lien de personnes issues de régions du savoir et de disciplines différentes.

On a beaucoup écrit sur le conceptualisme moscovite, et la recherche, focalisée sur les questions de poétique ${ }^{8}$ ou sur la place qu'il occupe dans l'histoire de l'art russe $^{9}$, a encore mal mesuré l'importance du travail conceptuel et philosophique des écrits et des œuvres d'Il'ja Kabakov ou des performances et des textes théoriques d'Andrej Monastyrskij et de son groupe Kollektyvnye dejstvija [Actions collectives], ainsi que la manière proprement philosophique dont ils ont construit et réfléchi leur œuvre théorique et artistique. À la recherche d'une forme d'autosuffisance théorique, ces éléments théoriques et langagiers sont présents à toutes les étapes de la création, notamment par l'abondance des discussions qui ont présidé à la constitution de ce milieu, mais aussi par la prédominance du texte théorique et du commentaire détaillé qui suivaient la conception de leur œuvre ${ }^{10}$. Cette excroissance théorique, cette exégèse infinie en constituent en quelque sorte le trésor caché, sur lequel il serait intéressant de lever un peu le voile. En cela, le groupe, tel qu'il s'est constitué initialement se distingue par exemple de l'école de Lianozovo qui rassemblait, elle aussi, des artistes et des poètes, mais n'affectait pas de dimension théorique et philosophique à leur œuvre ${ }^{11}$.

Les espaces réservés évoqués plus haut étaient l'occasion de rencontres avec des philosophes importants de la vie non officielle (le philosophe Merab Mamardašvili, le philosophe et spécialiste de la philosophie bouddhiste Aleksandr Pjatigorskij...). Il faudrait ici aussi rappeler en particulier l'intérêt renouvelé pour la philosophie religieuse russe et pour la philosophie orientale, qui constituent les deux pivots des révolutions et des mutations que connaîtra le conceptualisme moscovite au cours des années 1970. Des exposés présentés dans les ateliers, ou des discussions privées et libres ont constitué le terreau d'une formation à l'art de créer des concepts, ou à celui, plus apophatique, de cerner l'ineffable par le négatif.

Ce sont les philosophes eux-mêmes, en particulier ceux de la génération suivante qui ont trouvé inspiration dans le travail des conceptualistes. Deux philosophes

8. On peut mentionner ici le travail détaillé d'analyse de la logique poétique à l'œuvre dans les travaux du groupe KD (Kollektivnye dejstvija) par Sylvia Sasse, Texte in Aktion. Sprech- und Sprachakte im Moskauer Konzeptualismus [Textes en action. Actes du langage et de la langue dans le conceptualisme moscovite], Munich : Fink, 2003.

9. Ekaterina Degot', Russkoe iskusstvo XX veka [L'art russe du Xx ${ }^{\mathrm{e}}$ siècle], M. : Trilistnik, 2001.

10. Il faut rappeler ici quand même les travaux de Boris Groys, pionniers en la matière, qui pour la première fois ont mis en relief les similitudes entre le conceptualisme moscovite et ce que l'on pourrait nommer grossièrement French Theory. Voir Groys « Moskovskij romantičeskij konceptualizm » ou encore Mikhail Epstein « The Philosophical Implications of Russian Conceptualism », Journal of Eurasian Studies, 1, 2010, p. 64-71.

11. Voir le récit du peintre Vladimir Nemuhin qui évoque l'absence d'une «philosophie particulière » au sein du groupe. Il a été lui-même un membre fondateur de l'école de Ljanozovo avec Oskar Rabin et le poète Sapgir. G. Kizeval’ter, éd., Eti strannye semidesjatye, ili Poterja nevinnosti [Ces étranges années soixante-dix, ou La perte de l'innocence], M. : NLO, 2010, p. 193. 
russes (Mihail Epštejn et Boris Groys) ayant fait carrière par la suite aussi bien en Russie qu'en Occident ont chacun consacré des livres d'entretien et de réflexion sur les grands thèmes de l'œuvre de Kabakov ${ }^{12}$, et reconnaissent l'influence de son travail sur leur pensée. Cette pensée est apparue au moment opportun pour confirmer leur intérêt naissant pour le postmodernisme et forger l'idée d'une troisième voie spécifique dans le réseau d'idées et d'expérimentations propres au conceptualisme moscovite. Le lecteur peu familier des œuvres de Kabakov est surpris d'y trouver un artiste «faisant la leçon » à des philosophes confirmés, ainsi qu'une pensée en liberté capable d'improviser sur les thèmes les plus divers, démontrant ainsi à qui veut bien l'entendre que le travail accompli pendant de nombreuses années a été placé sous le sceau d'une philosophie spéculative aux dimensions élargies. Plusieurs exemples révèlent cette pensée en mouvement. Il faut tout d'abord considérer le contexte intellectuel des groupes d'artistes non officiels - ils jouissaient d'une somme importante de temps libre ${ }^{13}$ - , qui a engendré ce que les artistes ont appelé la troisième vie (tret'ja žizn'), vie faite de discussions informelles qui permirent une culture de la parole, de l'oralité au sein du groupe. À cette époque-là, on discutait de tout. Dans le large corpus théorique que Kabakov a constitué, indissociable d'une œuvre conçue comme processus, on trouve des commentaires de son travail, des entretiens, des réflexions assorties à des problématiques générales (les rapports textes-images, la condition de l'homme, la vie de la conscience, l'espace soviétique, l'imaginaire, la métaphysique de la lumière, etc.). Les textes philosophiques (sur la question du déchet par exemple) sont parfois des éléments d'une installation (l'installation « 10 personnages ») et sont la plupart du temps attribués à des personnages fictifs inventés par l'artiste. L'originalité de cette pensée prend forme dans la création de personnages imaginaires, singuliers et oubliés, prétextes au développement d'une idée philosophique, Kabakov parle à ce propos d'historiosophie ${ }^{14}$. L'intérêt pour ces vies minuscules s'inscrit dans l'arrière-fond de la littérature russe du xix ${ }^{\mathrm{e}}$, rappelant tout autant le personnage du manteau de Gogol' que les protagonistes des romans de Dostoevskij.

Kabakov étant un artiste reconnu et encore très actif sur la scène artistique internationale contemporaine, il faut remonter aux années cruciales de sa formation artistique, celles où domine encore la peinture. Cette première période du travail de Kabakov qui s'étend de la fin des années 1960 jusqu'en 1974 précède cette révolution philosophique et esthétique nommée conceptualisme moscovite. Nous voudrions ici remonter à certaines sources philosophiques du conceptualisme moscovite, notamment reprendre la question de la métaphysique comme expérience, afin de mieux comprendre le parcours et les idées philosophiques de Kabakov, mais aussi de mieux comprendre pourquoi des questions métaphysiques,

12. Groys, « Moskovskij romantičeskij konceptualizm »; Epstein, « The Philosophical Implications... ».

13. Voir à ce propos le témoignage de Kabakov dans un recueil de témoignages consacrés aux années 70, Kizeval'ter, éd. Eti strannye semidesjatye, p. 94-95.

14. Il’ja Kabakov, Teksty [Textes], Vologda : Biblioteka Moskovskogo Konceptualisma, 2010. 
ontologiques ont autant imprégné le champ artistique. Malgré la claire distance affichée par le groupe dès sa constitution au milieu des années 1970 vis-à-vis de la possibilité d'une réflexion métaphysique, celle-ci s'inscrit en filigrane tout au long de son histoire intellectuelle ${ }^{15}$. Ces éléments devraient nous permettre de mieux appréhender les révolutions et renversements théoriques, passés inaperçus, qui ont secoué le milieu artistique, et en écho à cela, la philosophie elle-même.

\section{Au cœur du conceptualisme : l'empreinte de la métaphysique}

Si l'on connaît bien aujourd'hui - au travers des nombreux travaux sur le sujet - les œuvres des artistes appartenant au mouvement conceptualiste, leur usage ironique des codes soviétiques, le renouvellement des supports et des techniques proprement artistiques (des Albums de Pivovarov et Kabakov aux installations totales de Kabakov en passant par la poésie élémentaire de Monastyrskij), inversement, la part proprement philosophique et théorique qui constitue la spécificité de ce mouvement est méconnue. Il est vrai qu'elle ne se présente pas sous les abords les plus évidents. Outre que les artistes du groupe ne se sont pas définis comme des philosophes, il faudrait ajouter ici que l'occurrence de noms de philosophes prestigieux dans leurs textes est plutôt rare. Il est tout d'abord essentiel de reconstituer par le biais des différents témoignages les lectures essentielles de l'époque (Šestov, Florenskij, Bahtin, Heidegger...). Il faut également retenir ici un geste et un rapport aux idées qui s'inscrivent dans l'histoire des idées philosophiques (le conceptualisme moscovite peut être ainsi lu comme appartenant à la fois à l'histoire russe des idées par son lien avec les traditions de l'Âge d'argent, du suprématisme, voire de la théologie orthodoxe), retenir aussi l'omniprésence de textes théoriques qui empruntent à la philosophie le goût du raisonnement rigoureux, l'esprit d'analyse, la création de concepts et qui témoignent de la présence silencieuse de la philosophie, comme une sorte de rayonnement invisible venant du cœur de la pratique artistique. L'élargissement des frontières des disciplines apparaît comme le marqueur d'un certain nomadisme libertaire de circulation des concepts permettant la transgression des strictes frontières des disciplines. Jusqu'en $1974^{16}$ il est difficile d'exposer pour le vaste réseau d'artistes non officiels, la pratique d'une

15. En témoigne notamment le récit autobiographique d'Andrej Monastyrskij - artiste et fondateur du groupe Kollektyvnye dejstvija - consacré à son voyage initiatique aux marges de son psychisme, un parcours mêlé de folie et de mystique. Son expérience consacre une large part à la spiritualité orthodoxe, notamment à la technique dite de la prière du cœur : Andrej Monastyrskij, Estetičeskie issledovanija [Recherches esthétiques], Vologda : Biblioteka Moskovskogo Konceptualisma, 2009.

16. 1974 est une date-clé : c'est l'année de l'organisation d'une exposition des artistes non-officiels en dehors de Moscou, exposition qui sera détruite, sur ordre de Brejnev, par des bulldozers. Même si la plupart des conceptualistes n'y étaient pas présents, il s'ensuivit un puissant sentiment de soulagement : une certaine visibilité était acquise, et la menace étatique s'était affaiblie : Il'ja Kabakov, 1960-1970... Zapiski o neoficial'noj žizni v Moskve [1960-1970... : Notes sur la vie non-officielle à Moscou], M. : NLO, 2008, p. 292. 
muséographie «sauvage », dans des laboratoires ou dans les appartements s'en trouve favorisée. Ainsi, plus les conditions de visibilité des artistes s'élargissent, plus le cercle des participants et les frontières du groupe s'étendent.

Loin de revendiquer des ambitions philosophiques, il est aussi caractéristique du travail des artistes conceptualistes de parodier ou d'imiter le discours philosophique dans leurs écrits, voire de mettre à distance la philosophie elle-même ${ }^{17}$, rejoignant ainsi certains autres gestes philosophiques non conventionnels qui tendent à faire sortir la philosophie de ses frontières habituelles (Heidegger, Wittgenstein). Le questionnement et l'interprétation de la réalité soviétique comme une sorte de gigantesque texte, au travers du décalage ironique, de la simulation, de la mise en espace de ses textes, apparaissent comme un des motifs centraux de la réflexion des artistes. Ils constituent l'un des éléments qui font du conceptualisme moscovite une philosophie non conventionnelle, une anti-philosophie, qui suppose comme prémisse une liberté par rapport aux disciplines et aux méthodes employées, ce qui autorise les artistes à sortir de leur domaine spécifique. Ainsi, en 1986, Monastyrskij consacre un texte entier à l'analyse sémiotique, mythologique, symbolique du VDNH interprété comme un espace sacré ${ }^{18}$. Monastyrskij qualifie la méthode de schizo-analyse, comme celle de Deleuze avec laquelle elle n'a que peu ou pas de similitudes. Il faut voir là un transfert conceptuel qui se réalise sous l'influence de certains philosophes proches du groupe comme Mihail Ryklin ou Boris Groys. Ce texte, par sa mobilisation de méthodes d'analyse et de références hybrides, exerce une fonction critique de démystification et de compréhension des effets symboliques de l'appareil idéologique soviétique au travers d'un cas concret ${ }^{19}$.

Malgré les formes ascétiques de ses pratiques, le conceptualisme moscovite affirme la libre circulation des textes et des idées, l'élargissement du cadre figuratif au texte, voire à l'espace tout entier, mais aussi entre en dialogue avec les autres œuvres, celles de Malevič entre autres ${ }^{20}$. Mihail Epštejn, qui a travaillé sur la dimension philosophique du conceptualisme moscovite, affirmait la place centrale et originale qu'y occupent les idées (y compris les termes et les concepts) :

That is why Conceptualism, as a philosophy, is so strongly connected with art: the idea is used in its aesthetic capacity, as a verbal statement or visual

17. Dans sa préface au dictionnaire des termes du conceptualisme moscovite - dictionnaire qui réunit l'ensemble des termes et des concepts ayant fait leur apparition au sein du groupe Monastyrskij rappelle que le mouvement propose une poïesis des concepts, ou encore une poétique de la philosophie, si quelque chose de tel peut exister : Andrej Monastyrskij, Slovar' terminov moskovskoj konceptual'noj školy [Dictionnaire des termes de l'école conceptualiste moscovite], M. : Ad Marginem, 1999, p. 6.

18. Même si le texte appartient à une période tardive, il est malgré tout emblématique des travaux et des recherches langagières du groupe.

19. Monastyrskij, Estetičeskie issledovanija, p. 7-19.

20. On peut penser ici à une œuvre emblématique de 1986 « La courte histoire de l'art contemporain ou La vie et la mort du Carré noir » de Nikita Alekseev, fondateur du groupe Kollektyvnye dejstvija. Il s'agit d'un récit irrévérencieux sur la grandeur et la chute du Carré noir, figurant ici le destin de l'art russe au $\mathrm{xx}^{\mathrm{e}}$ siècle. 
projection of idea as such, so that all its factual or practical extensions are revealed as delusions. ${ }^{21}$

Pour Epštejn, la mise en scène de l'idée ou du concept dans l'œuvre est censée faire apparaître le caractère relatif de toute idéologie et donc anéantir toute idée de connexion entre art et réalité, ainsi que dissiper certaines illusions quant à la réalisation concrète de l'idée ou du concept dans le réel, tendant ainsi à lire le conceptualisme moscovite comme un proche parent du nominalisme. Il n'est pas sûr que les choses soient si simples. En effet, le conceptualisme moscovite ne présente pas une histoire homogène, et il n'est pas certain que Kabakov n'ait pas porté à certains moments de son œuvre une position plus réaliste que nominaliste, notamment sous l'influence de la métaphysique qui parcourt son œuvre et constitue véritablement l'envers de ce conceptualisme déconstruisant ironiquement les universaux proposés par le discours totalitaire. Nous allons ainsi essayer d'atténuer la portée de la thèse nominaliste - qui est avérée pour les travaux plus tardifs - en examinant les principaux aspects de cette métaphysique « concrète » sous l'influence de laquelle ont été réalisés les premiers travaux de Kabakov.

\section{Une relation à toute épreuve : art et philosophie}

Cette philosophie, cet ethos philosophique qui met au centre et comme objet du travail artistique le sens et les limites du tableau, se laisse mieux dévoiler depuis la publication des Mémoires de certains membres du groupe ${ }^{22}$ ainsi que de celle, ambitieuse, des écrits théoriques des différents artistes dans la collection «Bibliothèque du conceptualisme moscovite » (dirigée par German Titov) ${ }^{23}$ qui jouera certainement un rôle important dans la compréhension du conceptualisme moscovite comme philosophie.

Si Boris Groys a été le premier dans les années 1980 à publier des textes de référence sur le sujet, textes qui mettaient en lumière la dimension philosophique du geste conceptualiste, on découvre désormais à la lecture des textes théoriques de Kabakov une pensée en liberté aux accents singuliers qui, sans être alourdie de références théoriques, approche de près ou de loin quelques grandes réflexions de la philosophie d'un Wittgenstein ou d'un Derrida, sans avoir été réellement en contact avec celles-ci ${ }^{24}$, si ce n'est sous l'influence de Boris Groys. La question de la langue et de ses usages, la réflexion sur la place centrale de la littérature dans la culture

21. Epstein, « The Philosophical Implications... ».

22. Voir Viktor Pivovarov, Vljublennyj agent [L'agent amoureux], M. : NLO, 2001 ; Kabakov, $1960-1970 . .$.

23. http://www.conceptualism-moscow.org/page?id=404

24. Il était parfois difficile de se procurer des ouvrages de philosophie française à l'époque, sans parler des traductions, quasi inexistantes (à quelques exceptions près: la traduction des Mots et des Choses de Foucault en 1976 par Natalja Avtonomova constitue une première dans le paysage intellectuel des années 1970). 
soviétique (la culture soviétique comme culture littérarocentrée et logocentrique), mais aussi une pensée fondamentalement habitée par des questions ontologiques sur la nature du collectivisme, sur le sens de l'adjectif total, et sur l'espace ontologique du tableau constituent une des sphères de réflexion importantes du travail de Kabakov. Les influences, parfois paradoxales de cette pensée, conjuguent des sources différentes, voire contradictoires : à la fois la philosophie poststructuraliste et la métaphysique ou philosophie religieuse ${ }^{25}$, d'où une certaine difficulté pour appréhender cet objet hétérogène à l'appellation antinomique de proto-postmoderne ${ }^{26}$. Cette pensée constitue l'une des facettes passionnantes de cette époque mais n'en est pas la seule. Il est nécessaire de bien prendre en compte que si, à la même époque, l'on bataillait ferme en Occident pour se débarrasser de la métaphysique, en Union soviétique on s'acharnait plutôt dans certains milieux à reconstituer le lien avec une tradition métaphysique perdue, d'où sa permanence sous diverses formes tout au long du travail de Kabakov ${ }^{27}$.

La réception du conceptualisme moscovite aux États-Unis - avec le concours du travail théorique effectué par le couple Viktor et Margarita Tupicyn ${ }^{28}$ - est essentiellement marquée par une lecture «postmoderne » du sens philosophique de ce mouvement et a, en quelque sorte, occulté ce qui se cachait sous la surface $\mathrm{du}$ linguistic turn ou encore du poststructuralisme. Nous pourrions reprendre le mot de Kabakov lui-même et de certains de ses amis artistes de métaphysique pour qualifier cet autre versant. Un esprit métaphysique a soufflé sur certains groupes artistiques de l'époque soviétique de la fin des années 1960 au début des années 1970 , rappelle Kabakov dans ses Mémoires ${ }^{29}$. Cette phase ou période sera suivie, selon un découpage précis, par l'apparition brusque d'une période dite « sociale ». Cette tendance métaphysique, qui vient en quelque sorte tisser le destin futur des années 1990 (la réception de Heidegger, le retour à la philosophie religieuse, etc.), est liée de manière très directe à la réflexion de ces artistes non officiels sur les voies à prendre pour leurs travaux, et s'inscrit aussi dans le contexte spécifique d'isolement et d'impasse qui caractérisait le milieu artistique de l'époque. Afin d'échapper à cette exiguïté et à cet étouffement qui prédominaient, la recherche d'un ailleurs, le besoin de s'échapper ( $L$ 'art de s'envoler est le titre d'un entretien

25. Kabakov est resté en amitié et en contact avec des peintres influencés par la philosophie religieuse comme Edvard Štejnberg, Mihail Švarcmann, et Vladimir Jankilevskij.

26. Le terme est inventé au cours d'un séminaire privé par le poète et artiste Dmitrij Prigov. Il désigne ici une pensée ayant renoncé complètement aux idées émancipatrices de l'avant-garde russe.

27. Certaines toiles de la période explicitement métaphysique de Kabakov (fin des années 1960) seront réutilisées sur un mode plus distant dans des installations plus tardives comme «L'homme métaphysique » (Metafizičeskij čelovek). Dans cette œuvre, il ne s'agit plus de l'expérience métaphysique subjective et singulière mais de celle d'un anonyme : Kabakov, Teksty, p. 224-229.

28. Viktor Tupicyn, Kommunal'nyj (post)modernizm [Le (post)modernisme communal], M. : Ad Marginem, 1998 ; Margarita Tupicyna, Kritičeskoe optičeskoe [L'optique critique], M. : Ad Marginem, 1997.

29. Kabakov, 1960-1970... 
de Kabakov avec le philosophe Boris Groys) se font sentir de plus en plus fort. De plus, l'influence d'artistes non officiels non liés au conceptualisme moscovite comme Mihail Švarcman ou Edvard Štejnberg, dont le travail trouvait son inspiration dans la philosophie religieuse, fut importante.

La pratique de la philosophie prenait ainsi la forme d'une inclination vers la transcendance et témoignait d'un intérêt vif pour des questions métaphysiques vidées de leur contenu théologique. Il ne s'agit donc pas à proprement parler de l'élaboration d'une théologie systématique, mais plutôt d'une mise en espace des idées, qui apparaît déjà comme une source féconde pour les travaux futurs, d'une expression d'une certaine profondeur, d'une interrogation ontologique sur ce qui, dans le tableau, apparaît sous l'influence de la métaphysique de la lumière empruntée à l'iconologie byzantine, etc. Nous allons donc essayer de reconstituer quelques morceaux de cette métaphysique « oubliée » au travers des Mémoires et des textes théoriques de Kabakov, mais aussi et surtout au travers de figures d'influence qui ont constitué un sol fertile d'inspiration pour ces artistes.

\section{Une métaphysique vivante}

Dans ses entretiens avec le philosophe Boris Groys, Kabakov rappelle qu'à la fin des années 1960 et au début des années 1970, les cercles artistiques de Leningrad et de Moscou ont été gagnés par un soudain intérêt pour les «problèmes de renaissance religieuse et de spéculation philosophique pure, en particulier pour la pensée philosophico-religieuse de la fin du xix ${ }^{\mathrm{e}}$ siècle $»^{30}$. Kabakov a activement participé à cette quête métaphysique de la présence de Dieu dans le tableau, c'est pourquoi il est nécessaire de faire la lumière sur les liens qui unissent ces deux dimensions.

Le compagnon de route et ami de Kabakov, l'artiste Viktor Pivovarov, qui ne s'est pourtant pas revendiqué comme philosophe («je ne suis pas un philosophe, mais un sensible $»^{31}$ ), a, lui aussi, donné une définition de ce que signifiait pour lui la métaphysique. Cela va nous servir de point de départ pour aborder la problématique :

Je ne suis pas philosophe mais artiste, et je comprends le mot «métaphysique » comme un artiste. Ce mot signifie pour moi la présence dans le tableau de l'autre. ${ }^{32}$

À cet autre, qui ici n'est, à proprement parler, pas défini, et qui n'est pas forcément à entendre dans un sens théologique, résonne le sens d'un appel vers un ailleurs, vers ce dont on ne peut pas parler, mais que l'on peut seulement essayer

30. Boris Groys \& Il’ja Kabakov, Dialogi [Dialogues], Vologda : Biblioteka Moskovskogo Konceptualizma, 2010.

31. Pivovarov, Vljublennyj agent.

32. Ibid., p. 84. 
de désigner, d'approcher, etc. On y décèle aussi un élément extérieur au tableau lui-même. Essayer de situer, de canaliser cet autre apparaît comme l'une des préoccupations majeures des artistes dans ces années-là. Il s'agit ainsi pour des artistes comme Kabakov de lever le voile sur de nouveaux mondes, et cette tâche de dévoilement, empreinte de philosophie religieuse, s'incarnera dans un ensemble d'œuvres, de discours, de positions théoriques autour de la question du vide, du blanc, qui appellent d'une part à la contemplation de ce qui ne peut pas se représenter, mais aussi à une forme d'expérience d'abandon de soi dans un espace nul, ce que Malevič appelle la $5^{\text {e }}$ dimension, celui de la toile blanche par exemple. Nous y reviendrons plus loin. Dans son article ${ }^{33}$, Boris Groys rappelle que les spécificités du conceptualisme moscovite tiennent à ce qu'il nous fait entrevoir par le langage de l'art les structures d'un autre monde, avec la possibilité d'y fuir sans réserve bien réelle. Cela pourrait bien être ici un héritage métaphysique s'exprimant dans un intérêt constant des artistes conceptualistes pour l'expérience mystique, même vidée de connotation religieuse. Il faut bien insister ici sur le fait que cette approche pratique et théorique de la métaphysique se rapporte chez les artistes de cette époque à une expérience existentielle forte qui est d'abord celle de la relégation hors des sphères officielles vers les espaces clos ou souterrains que sont les cuisines, les ateliers. Ce confinement vers l'intime se double d'une vie collective et amicale intense, comme celle que vécurent les artistes Kabakov, Pivovarov, Jankilevskij et Štejnberg à leurs débuts, avant même l'existence du conceptualisme moscovite.

Cette question de l'espace de vie, fait en quelque sorte écho à la question de l'espace du tableau qui connaîtra un développement fertile dans la suite de l'histoire du conceptualisme moscovite, avec un jeu autour des frontières et des limites du tableau. Viktor Pivovarov a rappelé l'influence importante de Vladimir Favorskij (1886-1964), peintre, théoricien charismatique, et professeur à l'Académie des Beaux-Arts de Moscou :

Le concept d'espace a été un concept-clé de la philosophie artistique de Favorskij. Rien d'étonnant à ce que dans le système soviétique claustrophobe et fermé, il fût nécessaire de chercher une porte de sortie. Pour Favorskij une telle porte se trouvait dans la compréhension métaphysique de tout objet comme espace. ${ }^{34}$

L'espace est aussi l'occasion pour certains artistes de faire l'expérience intime de la conscience et de la liberté qui lui est afférente. Cette expérience de retrait fait écho tant à « l'émigration intérieure » que traversèrent de nombreux intellectuels russes de cette période qu'à la philosophie existentielle du philosophe Mamardašvili qui tente de fonder une éthique construite sur l'effort et l'activité du cogito ou de la vie immanente de la conscience. Il ne s'agit pas d'examiner les structures empiriques

33. Groys, Moskovskij romantičeskij konceptualizm.

34. Viktor Pivovarov, O ljubvi slova i izobraženija [De l'amour du mot et de l'image], M. : NLO, 2004, p. 29-30. 
de cette expérience de l'espace, comme pourrait le faire une psychologie, mais de s'élever vers des hauteurs spéculatives nouvelles. La métaphysique conçue comme réflexion esthétique profonde, comme expérience spirituelle, ou encore comme méditation, apparaît comme le cœur idéal des pratiques artistiques de l'époque. L'espace de la conscience représente à certains égards un horizon essentiel dans les œuvres de Kabakov et de Pivovarov, se traduisant par la création d'un grand nombre des personnages fictionnels, destinés à incarner les idées et les expériences des artistes, retrouvant ainsi une vitalité nouvelle dans ces nouveaux mondes ou espaces.

Ces rapports entre art et métaphysique ne constituent pas véritablement un nouveau mélange des genres. Il ne faut pas remonter à une époque si lointaine, les années 1920, pour trouver des artistes bien connus comme Kazimir Malevič, Ljubov Popova ou encore Vasilij Kandinskij, qui ont véritablement mis au centre de leur activité artistique non pas seulement l'art en tant que discipline spécifique, mais aussi tout ce qui l'environne, l'élargissant ainsi à la réalité toute entière, voire à une sorte de destin cosmique. Cette fusion entre art et philosophie prenait la forme de l'élaboration d'une doctrine artistique aux dimensions trans-esthétiques. Le dépassement de la figuration à travers les compositions non objectives, la destruction de la tradition en vue d'une renaissance, un iconoclasme revendiqué, le tout dans une langue prophétique, spontanée (Malevič n'avait à proprement parler aucune culture philosophique), constituent un système radical dont l'influence souterraine sur cette génération d'artistes fut incontestable ${ }^{35}$. Le suprématisme, doctrine inventée par Malevič lui-même, est une utopie artistique qui cherche à triompher de l'objectivité de la représentation picturale pour atteindre une sorte de point zéro ou d'état suprême. Cette mise à l'épreuve de la peinture par le néant, que Malevič nomme le « rien dévoilé », révèle quelque chose qui rejoint ici la question religieuse, mais dans une manière différente de celle posée par le culte de l'icône ${ }^{36}$. Plus que d'une quête d'absolu, il faudrait parler ici de pure présence manifestée dans la neutralisation de toute représentation, dans l'absorption de toute couleur par un noir qui, en même temps, les contient toutes. La peinture ne représente plus, elle dévoile, elle manifeste ce qu'il y a au-delà des choses, au-delà des objets ${ }^{37}$. Le peintre Štejnberg pouvait ainsi évoquer le langage du suprématisme comme celui d'une langue métagéométrique qui se tiendrait au-delà des apparences.

Cette lecture mystique de la peinture proposée par le suprématisme s'est incarnée de manière évidente dans les œuvres du peintre Štejnberg proche de Kabakov à ses débuts, mais qui n'appartenait pas au groupe large des conceptualistes. Štejnberg a

35. Elle se poursuit dans la dernière génération d'artistes russes issus du conceptualisme russe. Ainsi Pavel Pepperštein, le fils de Pivovarov, a consacré plusieurs de ses œuvres au motif du carré noir de Malevič, qui constitue en quelque sorte l'horizon indépassable de l'histoire de l'art russe.

36. Gérard Conio propose au contraire une interprétation, très personnelle de l'avant-garde russe comme réaction à la modernité, arguant notamment que " l'icône a été la référence commune à toutes les recherches de l'avant-garde »: Gérard Conio, Les avants-gardes : entre métaphysique et histoire, Lausanne : L'Âge d'Homme, 2002, p. 19.

37. Pour une lecture phénoménologique originale de l'œuvre philosophique de Malevič, on pourra consulter le livre très phénoménologique d'Emmanuel Martineau, Malévitch et la philosophie, Lausanne : L'Âge d'Homme, 1977. 
développé dans son œuvre et sous l'influence du philosophe et mystique Evguenij Šiffers une peinture abstracto-spéculative, selon les mots de Kabakov lui-même, rappelant la peinture suprématiste, concernée avant tout par la lumière venant du fond du tableau. Il est en cela le digne héritier du suprématisme dans le paysage soviétique. Kabakov rappelle le rôle important joué par cette idée de lumière pour les débuts de sa création ${ }^{38}$, cela avant même les débats et les discussions sur la métaphysique qui surviendront plus tard. Dans ses notes consacrées à cette période du milieu des années 1960, il évoque l'expérience suivante :

À cette époque-là, quand je dessinais et je dessinais constamment, je ressentais de plus en plus l'action qu'exerçait sur moi une sorte de lumière, d'énergie lumineuse. De la profondeur de la feuille, dès que j'avais jeté quelques traits, une lumière de provenance inhabituelle agissait directement sur moi.$^{39}$

Cette lumière, qui vient d'en haut par opposition au génie humain qui, lui, vient d'en bas, intervient comme un élément étranger au cadre du tableau, elle vient de ses profondeurs. Dans cette approche métaphysique, avec laquelle Kabakov travaille, on trouve l'idée d'une hiérarchie à l'intérieur du tableau entre les éléments formels (la composition, les formes), et l'élément transcendantal, ou énergétique, pour reprendre un concept qui se trouve au cœur de la tradition orthodoxe ${ }^{40}$. L'idée de hiérarchie culturelle est très importante dans les premiers travaux de Kabakov, car elle permet de séparer l'authentique de l'inauthentique, le futile du nécessaire, la vérité du mensonge, réduisant en conséquence l'importance de ce qui est figuré au centre du tableau. Les figures ou les motifs peuvent apparaître parfois comme un obstacle à la venue de cette lumière bienfaisante (blagoj svet). On trouve une illustration remarquable de cette notion d'obstacle dans la série de toiles blanches réalisées en 1974 et intitulées Na kraju [au bord], en bordure desquelles sont dessinés de petits motifs, évoquant ici le rétrécissement du figuratif au profit de l'essence même du tableau, sa lumière ${ }^{41}$; ces détails, comme inscrits en marge du tableau, apparaissent comme indifférents par rapport à ce centre à partir duquel irradie la lumière. Kabakov décrivait ainsi cette série de toiles :

From behind the entire surface of the painting an even, warm flooding, sort of granular light moves toward me, or better said, streams. I feel that it is coming

38. Cette expérience de la lumière sera aussi très forte chez des artistes proches comme Erik Bulatov, ou Viktor Pivovarov.

39. Kabakov, 1960-1970..., p. 28.

40. Sur la notion d'énergies divines incréées, il est possible de remonter encore plus loin, à la mystique byzantine notamment, et en particulier à l'œuvre de saint Grégoire de Palamas, dans laquelle Dieu est identifié à un soleil éternel dont les rayons ont la capacité de toucher l'homme qui s’y exerce : J.-Y. Lacoste, éd., Histoire de la théologie, P. : Seuil, 2009, p. 134.

41. Sasse rappelle dans son travail combien Kabakov s'inscrit à la fois dans la tradition byzantine et celle de Malevič, puisqu'il « comprenait le blanc comme principe suprême », Sasse, Texte in Aktion, p. 86. 
from the infinite "far away," it envelopps me and disappears behind my back, not changing its power or energy. ${ }^{42}$

On est frappé par l'empathie qui émane de cette description. Les données primordiales de cette expérience sont ainsi assurées par la médiation de l'œuvre, à laquelle est attribuée une fonction de dévoilement de cette énergie, élément ineffable, chaud, rassurant. En plus d'être l'expérience d'un jaillissement, il faut insister sur le fait que cette métaphysique est une expérience (pereživanie) émotionnelle, physique, qui prend sa source dans le climat spirituel intense de l'époque ${ }^{43}$. Dans les travaux plus tardifs de Kabakov, la métaphysique va subsister, mais sous d'autres formes. On retrouve ainsi la présence d'une certaine forme de métaphysique dans les Albums ${ }^{44}$ que Kabakov consacra à des vies de personnages fictifs : dans un de ses articles, Šiffers les désigne comme « les cahiers métaphysiques de Kabakov ». Les Albums mettent en scène un parcours qui mène de la perception intérieure vers le vide, le rien, la mort, etc. Tous les Albums de cette série intitulée « 10 Personnages » s'achèvent sur une case blanche. Pour la tradition culturelle russe, rappelait Šiffers dans une conférence, l'œuvre est un « chœur de voix ». Le croisement, le bruit assourdissant des voix est un problème philosophique et existentiel qui va occuper constamment Kabakov dans son travail, des Albums jusqu'aux installations des années 1990. À propos de l'origine du projet des Albums, il écrivait son désir de voir tout ce concert assourdissant de voix, ressenti étant enfant comme une coupure d'avec le monde extérieur, apaisé par l'extraction en détail de chacune de ces voix, de leur mise en inventaire. Ce désordre oppressant, associé à une posture et à un questionnement ontologique et existentiel (où se trouve le sujet, une fois le concert de voix détaillé ?), illustre un thème essentiel de la période des années 70, l'exploration intérieure. Ce théâtre de la conscience était caractérisé ainsi par Kabakov dans un texte consacré aux Albums :

Ainsi, « les thèmes-images », ayant servi à la confection des « 10 personnages », sont précisément les thèmes de ma conscience qui, maintenant depuis déjà longtemps, peuvent être présentés comme les «mythèmes » fondamentaux de condensés douloureux, de complexes, de névroses et même d'hystéries, je ne sais pas comment les appeler plus précisément. ${ }^{45}$

La mise en espace de ces motifs passera par la création de personnages à même de les porter, dont le thème sera le contenu unique de toute son existence. Cette cristallisation d'une idée-personnage, comme nous pourrions l'appeler, naît, se développe

42. Amei Wallach, Ilya Kabakov: The Man Who Never Threw Anything Away, with comm. by I. Kabakov, New York : H. N. Abrams, 1996, p. 148.

43. Climat spirituel que l'on retrouve autant à Leningrad qu'à Moscou. Il prend forme dans la publication en samizdat de la philosophie religieuse russe, et apparaît plus généralement comme un désir de connexion avec la tradition. Ibid., p. 44.

44. Il s'agit d'une série de feuilles illustrées mêlant texte et image dans un rapport particulier, dont on dira en substance que le texte constitue une illustration à part entière, à égalité avec l'illustration elle-même. Kabakov entame sa première série d'Albums « 10 Personnages » en 1972.

45. Kabakov, Teksty, p. 17. 
et meurt. La vie d'une idée, apparaît comme une manière de mettre en scène l'intériorité de la conscience, unique espace possible pour ce que Kabakov nomme «l'historiosophie du personnage », une manière de suivre le destin de cette idée et de cette conscience sous le plus de points de vue possibles, au travers notamment de commentaires extérieurs qui objectivisent la vie de cette idée. Il y a donc bien une évolution, un changement d'échelle d'une métaphysique de la lumière, comme expérience existentielle, vers la description soigneuse de la vie de personnages fictifs mettant en scène le théâtre de la conscience d'un sujet tourmenté. Tous ces éléments s'articulent bel et bien dans des interrogations philosophiques exposées dans des œuvres d'art.

\section{Un acteur occulte : Evguenij Siffers}

Il nous faut revenir maintenant sur Evgenij Šiffers, personnage singulier qui joua un rôle essentiel dans la transmission de ce souffle métaphysique. Formé d'abord au théâtre à Saint-Pétersbourg, Shiffers est un penseur et un réalisateur au parcours original. Il a peu voyagé, rempli des montagnes de carnets, rédigé de nombreuses notes de lectures et entretenu de longues correspondances avec d'autres penseurs ${ }^{46}$ mais n'a presque rien publié de son vivant. En 1967, par suite d'un conflit avec Lenfilm autour d'un scandale causé par son premier film, il part s'installer à Moscou où il commence à fréquenter les milieux artistiques non officiels. Il trouve un public ouvert à son enseignement et à son savoir érudit. La publication récente à Moscou de trois tomes des œuvres et des notes de Šiffers ${ }^{47}$ permet de se faire une idée de la variété de ses lectures et de l'ampleur de son projet de mise en lien avec la tradition. L'intensité avec laquelle il «prêchait » son savoir laisse deviner ou apprécier l'influence qu'il a eue sur le premier cercle des conceptualistes, Vladimir Jankilevskij, Edvard Štejnberg, Viktor Pivovarov le critique, entre autres. Nous allons donc essayer de mettre en avant certains traits de sa pensée et de les relier aux premières expériences métaphysiques de Kabakov.

La pensée de Šiffers est inspirée par la philosophie de Pavel Florenskij, mais aussi par les écrivains de l'Âge d'argent, la philosophie existentielle de Šestov ou des philosophes conservateurs du xix ${ }^{\mathrm{e}}$ siècle comme Konstantin Leont'ev. Ces différentes lectures, assorties d'une réflexion spéculative sur les formes artistiques nationales partant d'une définition organique de la culture, serviront son travail sur ce qu'il nomme «l'intensification de sa conscience nationale. Pour Šiffers, la culture forme un tout qui préexiste à ses parties, dont le but est la recherche du sacré, l'homme devant tendre vers la sainteté. Cette réflexion spéculative, non dénuée de dimensions concrètes, passant notamment par la pratique spirituelle comme la

46. Il a été très lié à un philosophe membre du Cercle méthodologique de Moscou, en la personne d'Oleg Genisareckij.

47. Evgenij Šiffers, Religiozno-filosofskije proizvedenija [CEuvres religieuses et philosophiques], M.: Russkij institut, 2005. 
méditation, ou la prière, ne constituera pas un cas isolé au sein des communautés non officielles ${ }^{48}$. L'intérêt donc pour la lumière et sa métaphysique, pour le sacré, pour le tableau comme icône et non comme toile sont autant de thèmes qui furent intensifiés et développés par l'intermédiaire de Šiffers, lequel entretint également une correspondance avec Kabakov et d'autres artistes, autour notamment du rapport entre culte et art. Il participera par exemple aux festivités des cent ans de Malevič organisées dans l'atelier de Kabakov en 1977 et contribuera à imposer une interprétation spirituelle de l'œuvre du fondateur du suprématisme tout en s'en distanciant ${ }^{49}$. En fondant son approche sur un lien étroit retrouvé avec la culture russe, lien détruit par l'événement révolutionnaire, Šiffers construit un discours essentialiste de reconstruction de l'identité culturelle :

Il a été dit, disons par Puškin, que tout notre savoir était tiré de livres étrangers, que nous n'avions pas de langage métaphysique propre, avec lequel nous pourrions penser la culture, il nous faut pourtant le faire. Où pouvons-nous le prendre ? Ici s'éclaire le rôle existentiel et ontologique du poète et de son génie créateur. ${ }^{50}$

Trouver sa propre langue, construire un monde à soi, telles étaient les tâches de nombreux artistes et intellectuels de cette génération qui ne pouvaient s'identifier totalement ni à l'institution en place, ni aux artistes de la génération précédente. La réduction progressive des formes au sein du travail de Kabakov, la tendance vers une certaine forme de minimalisme, mais aussi l'émergence du texte sur le même plan que la figuration, sont des éléments qui aident à la circulation et à la diffusion de cette lumière, rejoignant ainsi certaines idées spiritualistes inspirées de Šiffers. À propos de la sainteté, Šiffers affirmait, dans une conférence donnée devant le Cercle méthodologique de $\mathrm{Moscou}^{51}$, que la culture russe recherche la sainteté, prenant comme inspiration ce que Dostoevskij appelle l'hagiocratie, le pouvoir du sacré. Dans cette recherche pratique de la sainteté, qui pouvait s'incarner autant dans la peinture que dans la pensée, la méditation, la poésie, se donnent justement pour tâche la transmission de cette lumière. Les formes d'ascétisme qui caractérisaient la vie au sein du conceptualisme moscovite, Pivovarov parle d'ascétisme sévère $^{52}$, trouvent en partie leur source dans cette métaphysique radicale qui place la tâche de l'artiste à l'égal de celle du moine ou de l'ascète. Cet intérêt pour la vie spirituelle et ce rassemblement en petite communauté constituent une phase importante de l'histoire de la philosophie de ces années-là, une sorte de troisième

48. On peut penser sur un mode plus artistique et concret, à la poésie d'Andrej Monastyrskij construite sur le modèle du mantra, ainsi que son intérêt constant pour les religions orientales par exemple.

49. Pour Šiffers, le suprématisme est une usurpation du christianisme.

50. Evgenij Šiffers, Religiozno-filosofskije proizvedenija [Euvres religieuses et philosophiques], M.: Russkij institut, 2005, p. 450.

51. Svetlana Tabatchnikova, Le cercle méthodologique de Moscou (1954-1989) : une pensée, une pratique, P. : Éditions EHESS, 2007.

52. Pivovarov, O ljubvi slova, p. 65. 
réveil philosophique comme l'appelle M. Epštejn ${ }^{53}$, et ne concernent pas seulement ce cercle-là. Il existait aussi le Južinskij krug, cercle occultiste et traditionnaliste de E. Golovin et Yu. Mamleev qui ont influencé l'inspirateur du néo-eurasisme ${ }^{54}$ Alexandre Dugin. Celui-ci sera un acteur important de la révolution conservatrice que la Russie connaîtra dans les années $1990^{55}$.

Si les positions de Šiffers se sont radicalisées avec le temps en un nationalisme russe radical et délirant, purifié de toute influence occidentale, il aura permis à ces artistes, selon Boris Groys ${ }^{56}$, de trouver un style et une posture qui ne soient pas uniquement l'imitation de ce qui se faisait en Europe. Ainsi un artiste comme Štejnberg rappelle dans ses souvenirs combien il était important de développer son propre style, mais aussi combien Šiffers, lui-même, dont il était très proche l'avait aidé à comprendre ce qu'il faisait, à mettre en mots l'essence de sa peinture ${ }^{57}$. Pourtant, l'émergence d'une nouvelle tendance, dont les artisans du progrès et de la révolution en cours prendront rapidement les commandes, va annoncer une recomposition du champ artistique, suscitant parfois parmi les autres artistes un sentiment d'incompréhension et de regret. Dans ses entretiens avec Groys, Kabakov évoque la déception de Šiffers lorsqu'il découvre les œuvres postérieures aux Albums dans lesquelles sont effacées toutes traces d'une dimension sacrée ou prophétique, ou n'en conservent tout du moins que le caractère référentiel : « Tu te tenais pourtant haut dans la hiérarchie spirituelle... ${ }^{58}$.

Ainsi, il est nécessaire de comprendre la fin de cette période métaphysique qui coïncide par ailleurs avec l'événement de « l'exposition aux bulldozers » $(1974)^{59}$ comme constituant symboliquement l'épreuve de la visibilité pour des artistes ayant longtemps vécu dans la peur, et le début d'une certaine reconnaissance. La fin donc de cette métaphysique est à prendre au sens plein du terme puisqu'elle signifie véritablement la sortie du monde intérieur, que cela se manifeste par l'occupation de grands espaces naturels dans les performances organisées à l'extérieur par le groupe Kollektyvnye dejstvija, mais aussi par l'ouverture du cercle des artistes

53. Mihail Epštejn http://www.bu.edu/wcp/Papers/Cont/ContEpst.htm. L'hypothèse la plus intéressante de l'article souligne les ambivalences propres à cette libération d'une métaphysique radicale dans les années 1970 qui a permis au cours de la perestroïka l'apparition d'un discours extrême occupant soudainement le centre de la vie politique et intellectuelle. Cette libération de la boîte de Pandore aurait en quelque sorte eu comme conséquence l'établissement d'une voie royale pour tous les extrémismes que connaît et qu'a connu la société russe postsoviétique.

54. Marlène Laruelle, La quête d'une identité impériale : le néo-eurasisme dans la Russie contemporaine, P. : Petra, 2007 ; l'ouvrage est important pour la compréhension de la révolution conservatrice que ces cercles appellent de leurs vœux.

55. Réunis dans l'appartement de Jurij Mamleev, écrivain, auteur de romans étranges mettant en scène une Russie soviétique magique, occulte. Voir http://eurasia.com.ru/versia.htm.

56. Correspondance privée avec B. Groys, 21 avril 2011.

57. Edvard Štejnberg, « Ja sformirovalsja v šestidesjatye » [J'ai été formé dans les années 60], in Kizeval’ter, éd., Eti strannye semidesjatye, p. 351.

58. Groys in Groys \& Kabakov, Dialogi, p. 71.

59. http://back-in-ussr.livejournal.com/138915.html : la polémique sur internet au sujet des conditions de travail des artistes en URSS. 
non officiels initiaux à d'autres sphères : celle de la poésie notamment ou de la philosophie. Šteinberg évoque ainsi son sentiment à propos de cette époque :

Lorsqu'ont commencé tous ces jeux néo-avangardistes, ça m'a tout de suite déplu. Il'ja Kabakov par exemple, était un métaphysicien, Šiffers l'a d'ailleurs écrit dans son article « les Albums de Kabakov » [Les cahiers métaphysiques de Kabakov], d'autant qu'ils se trouvaient à l'époque dans le même groupe, mais ensuite Il'ja est tombé sous l'influence du sots-art..$^{60}$

Au-delà d'un constat exagéré (Kabakov n'a jamais été sous l'influence du sots-art en tant que tel, il s'en est toujours distingué, cherchant lui aussi sa langue propre et affichant une certaine constance thématique), on perçoit ici immédiatement que derrière la critique de Štejnberg se cache une distinction entre les jeux peu sérieux de certaines activités artistiques et la métaphysique comme tâche supérieure, élevée. Il est possible ainsi de reconstituer le sentiment intense d'appartenance à une élite que pouvaient nourrir certains artistes à l'idée de faire partie d'un cercle de réflexions aux tâches aussi importantes.

Ainsi le retour aux sources métaphysiques - dont l'atmosphère spirituelle intense et concentrée a, selon Kabakov, porté son influence jusqu'à une période postérieure à la fin de ce souffle métaphysique ${ }^{61}$ - aura été un épisode décisif dans la constitution d'un champ artistique autonome et fécond.

\section{Le nouveau champ des signes}

Dès le début des années 1970, une autre tendance importante prenait forme au sein même du conceptualisme moscovite. Elle apparaîtra plus tard comme celle retenue par les critiques lors de la réception du conceptualisme moscovite à l'étranger, notamment sous l'impulsion du mathématicien et théoricien de l'art Viktor Tupicyn et de son épouse Margarita, tous deux ayant émigré à New-York. Connue sous le nom de sots-art, cette branche du conceptualisme réunit des artistes aussi différents qu'Erik Bulatov ou Vitalij Komar et Aleks Melamid. Le terme exprime la fusion des mots « réalisme socialiste » et « art », rappelant ainsi le pop-art et, dans le même temps, marquant ses spécificités dues à la différence entre les contextes américain et russe. Il s'agit en substance d'une tendance artistique qui emprunte les signes idéologiques de la propagande soviétique (ses slogans, ses affiches, ses icônes), mais utilise aussi les codes du système esthétique en vigueur à l'époque, pour les tourner en dérision, les désacraliser (Komar et Melamid), ou pour en montrer les limites et les frontières (Erik Bulatov). À un système qui se présentait comme absolu, achevé, sans failles, soutenu par une sorte de signifié transcendantal (la doxa marxiste), les artistes répondirent par une sorte de révolution du

60. Štejnberg, « Ja sformirovalsja v šestidesjatye », p. 354-355.

61. Notamment dans la constitution de ce que Kabakov appelle dans une expression difficile à rendre, « l'art du milieu » (iskusstvo sredy), Kabakov, 1960-1970..., p. 92. 
signifiant, dans laquelle ces œuvres majestueuses détournées de leur socle d'énonciation initial apparaissaient comme de simples œuvres parmi d'autres ${ }^{62}$. Jouant du décalage et de l'ironie, à l'image de la reproduction grandiloquente de certains slogans sur les toiles de Bulatov, cette pratique artistique fonctionnait comme une véritable machine à mettre les systèmes idéologiques sur le même plan.

Si donc, dans un premier temps, le sots-art prend son envol, la révolution véritable, la transformation du champ artistique interviendra avec l'apparition du groupe artistique KD, Kollektivnye dejstvija [Actions collectives] ${ }^{63}$, qui propose des performances collectives, entamées en 1976 et constituant le cœur d'action du conceptualisme moscovite comme communauté d'artistes. Ces actions collectives portent leur attention sur les structures de perception, la constitution du sens au sein d'une communauté, son énonciation, mais aussi sur la désautomatisation des réflexes quotidiens. Elles constitueront un laboratoire influent pour la constitution d'un nouveau champ artistique, et serviront l'invention d'un genre nouveau sur le sol soviétique.

Faisant sienne l'expérience de l'étonnement philosophique, cette philosophie appliquée déplacera en profondeur les problématiques ontologiques initiales (espace du tableau, métaphysique de la lumière, etc.) vers une sémiotique sui generis, dont le discours théorique rigoureux constitue un modèle du genre. Monastyrskij nomme ce travail poésie de la philosophie ${ }^{64}$, il est possible de la voir comme une philosophie du langage à part entière, dans le droit sillage de la philosophie de Wittgenstein. En réunissant autour d'elle les artistes non officiels, toutes tendances confondues, cette pensée contribuera à construire le conceptualisme moscovite comme une communauté d'intérêts communs et d'échange d'idées.

\section{Une métaphysique revue et corrigée}

Il faut maintenant ajouter quelques mots au regard de l'évolution de la pensée de Kabakov qui prendra une direction sensiblement différente, au gré de l'évolution des supports artistiques investis par l'artiste. Au cours des années 1980, celui-ci

62. Les artistes Komar et Melamid ont par exemple signé de leur nom d'artiste de grands drapeaux rouges portant des slogans à la gloire du communisme. Cette signature nouvelle transforme le code initial grandiose et triomphant en une simple énonciation singulière, faisant ainsi apparaître cette propagande de vérité comme une phrase énoncée par un sujet artiste, lui faisant ainsi perdre toute valeur de vérité ou d'effectivité.

63. Dans le même temps, on trouve aussi dans l'histoire interne du conceptualisme un événement qui marque la rupture définitive d'avec la métaphysique : une altercation se produit entre Groys et Šiffers après une exposition de Štejnberg en 1978. Elle marquera la fin du profond lien d'amitié qui unissait le petit groupe de Kabakov, Pivovarov et Jankilevskij : Šiffers Religiozno-filosofskije proizvedenija, p. 583.

64. Par poésie de la philosophie, Monastyrskij pense au travail théorique de production de concepts présenté dans son dictionnaire, un effort tendant non vers la création de concepts plus opératifs, plus réels, mais vers une réduction de la philosophie à un jeu de langage (Wittgenstein n'est jamais loin), à son envers imaginaire, dont les règles se redéfinissent dans l'œuvre conçue comme processus (en cela les artistes de cette époque sont bien les héritiers de l'art conceptuel) : Monastyrskij, Estetičeskie issledovanija, p. 6. 
commence à agrandir l'échelle de ses travaux, à développer ce qu'il appelle installation totale, qui diffère de l'installation en vigueur dans le monde occidental. Dans ce travail, entrepris après son émigration, Kabakov soutient l'idée d'une différence de nature entre les deux mondes, soviétique et occidental, entre deux manières de faire de l'art, mettant au centre de son travail la question de l'archive et du déchet. Son concept d'installation totale et son caractère d'expérience vécue redimensionnent considérablement son travail, cherchant à donner une portée globale de réflexion sur le destin du « cosmos » russe. L'artiste entreprend de réutiliser, de recycler des œuvres plus anciennes, constituant ainsi à travers la conservation de sa propre œuvre préservée de la disparition, une archive «brute » de la période soviétique tardive. Cette manière de faire des mondes nouveaux, des mondes imaginaires hantés par l'archive impossible de la vie anonyme soviétique, apparaît toujours comme une reconstruction du monde ancien.

Ayant définitivement quitté la métaphysique au sens quasi religieux et spirituel qu'il lui avait donné dans ses premiers travaux, Kabakov développe une sorte d' « anti-métaphysique », métaphysique du vide ou du déchet ${ }^{65}$, dans laquelle l'espace soviétique dans sa totalité est vu comme une catégorie philosophique ${ }^{66}$. En même temps qu'il agrandit la dimension de ses œuvres, il élargit l'objet de son intérêt à la culture soviétique dans son ensemble, préservant une forme de questionnement ontologique sur la nature des modes d'existence sous le régime soviétique dont il trouve les grands concepts dans la vie collective (kommunal'nost'), instanciée par l'invention d'un langage artistique nouveau. Tout comme le Cercle méthodologique de Moscou simulait dans des « jeux » grandeur nature des questions sociétales d'importance majeure, Kabakov reconstitue, produit littéralement un univers en simulation sous ses aspects pluriels, par la reproduction de documents officiels, le mélange de discours de provenances diverses, gardant toujours en tête qu'il s'agit d'une reconstruction ironique et subjective et non de l'objet réel. Ce gigantesque simulacre, cette exploration de l'inconscient collectif marquent ainsi une rupture importante avec les premiers travaux, puisque le questionnement ontologique est préservé dans une exploration non seulement de l'inconscient, mais aussi des structures de la réalité imaginaire elle-même, celle d'une Union soviétique reconstituée librement et comme transfigurée. Selon Kabakov, tout simulacre doit conduire à l'acceptation du caractère relatif de tous points de vue, et c'est dans la multiplication de ceux-ci et leur effeuillage soigneux que se trouve la clé d'une compréhension plus fine et nouvelle de nos mondes multiples, ceux de la conscience et de l'imaginaire. Opposé à toute chosification du réel, Kabakov préfère à cette idée la libération de l'imagination, faisant ainsi de son art une sorte de phénoménologie, prétexte pour un voyage en profondeur dans les possibilités multiples de la conscience.

65. Nous reprenons ici la formule du philosophe Mihail Epštejn.

66. Epštejn évoquait cette phase de l'œuvre tardive de Kabakov ainsi : « Kabakov's thought is remarkable in that it focuses almost entirely on unique features of Soviet civilization and interprets them as general philosophical categories. The central category of his world-view might be called emptiness, or void, which he views as fundamental to Soviet reality » : Epstein, «The philosophical implications... », p. 67. 


\section{En guise d'épilogue : l'avenir d'un passé}

Ce parcours dans la pensée soviétique que nous venons d'esquisser n'est qu'un des aspects de la révision que son histoire suscite. Il met en lumière la situation particulière du groupe des conceptualistes qui, plus que d'autres groupes, aura vécu au rythme du samizdat et du tamizdat, concentrant son énergie intellectuelle sur la détermination d'une ligne théorique, d'un geste philosophique de questionnement radical sur les sens possibles de l'art, sortant ainsi constamment du cadre fixé par l'idéologie officielle d'État. De plus, le conceptualisme moscovite a été mis en forme philosophiquement par sa conception de l'œuvre comme processus, poiesis de concepts et comme projection d'idées, intégrant à son canon tout un univers de textes et de réflexions philosophiques, mais aussi par la présence au sein du groupe de philosophes confirmés (Evgenij Šiffers, Oleg Genisareckij, Boris Groys, Mihail Ryklin) et par l'assimilation de la pensée occidentale. Opposant les années 1970 aux années 1960, le sculpteur Boris Orlov évoque l'élan des artistes non officiels à comprendre la culture philosophique occidentale comme celui d'un esprit d'assimilation caractérisé comme audacieux, agressif et non scolaire. Les artistes de cette époque, coupés de l'espace international de circulation des idées, se sont sentis le centre du monde, et conscients de ce qu'ils pouvaient incarner dans le champ intellectuel ${ }^{67}$. Cette configuration intellectuelle particulière a permis l'affranchissement de la philosophie de son cadre institutionnel, contribuant à façonner des thématiques qui seront essentielles pour la génération postsoviétique, notamment l'idée d'une « philosophie aux limites ${ }^{68}$ développée par le philosophe Valerij Podoroga, manifeste de l'école d'anthropologie analytique, autre signe d'un renouveau philosophique. Par son libre maniement des concepts, son nomadisme intellectuel et son anti-dogmatisme, le conceptualisme moscovite s'est nourri des forces intellectuelles de son temps, il s'est construit comme une pensée en marge, contribuant ainsi à donner l'exemple d'une recherche vivante de nouvelles voies pour la création et la connaissance.

emanuel.landolt@gmail.com

Université de Saint-Gall, Suisse

mmaiatsky@gmail.com

École des hautes études en sciences économiques, Université d'État, Moscou

67. Orlov in Kizeval’ter, éd., Eti strannye semidesiatye, p. 206-207.

68. Cette pensée philosophique qui se dessine aux extrêmes d'une expérience culturelle dominante, ne prétend nullement à l'occupation d'un espace dans un centre nouveau, elle indique plutôt à partir de quoi se forme le tissu idéel et culturel d'une époque. Cette dernière découvre des fibres de pratiques de pensée diverses, s'entrelaçant librement les unes avec les autres en d'hasardeuses et capricieuses arabesques, toujours aux « limites », c'est-à-dire qu'elles semblent s'opposer à toute tentative de simplification violente de leur texture : Aleksandr Ivanov, « Entretien avec Valerij Podoroga et Mihail Jampolskij », Ad Marginem 93, Ežegodnik Laboratorii postklassičeskih issledovanij IF RAN [Annuaire du Laboratoire de recherches postclassiques de l'Institut de philosophie de l'Académie des sciences russe], M. : Ad Marginem, 1994. 